\title{
Floristic diversity, biological spectrum of lower Tanawal, KP, Pakistan
}

\author{
Adeela Bibi, Zafar Iqbal", Ghulam Mujtaba Shah, Manzoor Hussain and Inayat UR \\ Rahman \\ Uman National University of Horticulture, Uman, Ukraine \\ Department of Botany, Hazara University Mansehra-21300, Khyber Pakhtunkhwa, Pakistan
}

\author{
E-mail: drzafar.hu@yahoo.com
}

Received: 14.11.2019. Accepted: 05.12.2019

\begin{abstract}
Field surveys were carried out to evaluate the floristic diversity and biological spectrum of Lower Tanawal, District Abbottabad in different seasons during 2016-2018. This study area was divided into 80 stands on the basis of physiognomy of the vegetation. Life form classes and leaf spectra were classified following Raunkier's methodology. In total, 286 plant species belonging to 86 families were recorded, out of which herbaceous growth form dominated the study area with 187 species, followed by shrub with 54 species. Among all 86 families, Asteraceae was found to be the most dominant family with 25 species, followed by Fabaceae with 24 species and Rosaceae with 20 species. Based on life form classes, Therophytic life form was the leading class with 113 species (39.5104\%), followed by Nanophanerophytes with 45 species (15.7342\%). In leaf size spectra, Microphylls dominated the flora with 88 species (30.7692\%), followed by Mesophyll with 81 species (28.3216\%). This study shows the complete floristic composition that may be important as reference work for future ethnobotanical, ecological and conservational studies.
\end{abstract}

Key words: The floristic composition; Biological spectrum; Lower tanawal; Abbottabad; Pakistan

\section{Introduction}

Flora is a priceless donation of nature upon which the mankind always relies (Khan et al., 2013). Flora comprises the total plant species of any specific geographic region, which are characteristic of a geological period or in an ecosystem (Durrani et al., 2005). Nonetheless, the most useful source of botanical information of a particular area is its floristic checklists. Since, floristic composition is a decent floristic marker hence, any sort of changing floristic composition in various endogenous milieus highlights the presence of various environmental variables, prompting entomb and intra-variations (Safidkon et al., 2003). Floristic structure of a specific territory provides fundamental yet assorted information about the plant dissemination; and the motherland has diverse landscapes, topographies, and biological/ecological zones, which bolster distinctive floristic composition (Masroor, 2011). To contemplate the vegetation of a territory, it is essential to oversee the plants' life form, which is an indicator for both micro and macroclimate (Shimwell 1971). As leaf sizes and shapes (physiognomy) connects unequivocally with moisture and temperature from worldwide to neighborhood scales (Bailey and Sinott, 1915). Literature survey reveals that the study area is still virgin as no work has been done on biological spectrum in Pakistan (Shaheen et al., 2016). Thus, the present study was designed to document the floristic composition and biological spectrum of this unexplored area of the northern Pakistan.

\section{Materials and Methods \\ Study area}

The Lower Tanawal situated in District Abbottabad, Pakistan which lies between the latitudes $34^{\circ} 12^{\prime} .328,34^{\circ} 15^{\prime} .761 \mathrm{North}^{\prime}, 73^{\circ}$ 09' 398", 73.03'. 316 East. The area occupies the intervening area between the Mansehra basin in the north and the Haripur basin to the south, adjacent and NE of the Tarbela Dam reservoir. Lower Tanawal is located in District Abbottabad Khyber Pakhtunkhwa province, located towards west of Abbottabad. The altitudinal range of Lower Tanawal is from 2396 feet at Chamhad to 6742 feet at Karagali. Lower Tanawal is a component of many small and big villages almost adjoining each other. Lower Tanawal is located in a beautiful valley which is surrounded by large forested mountains on three sides. The boundary of Lower Tanawal area start from the village Paswal which is away from Abbotabad on the distance of $10 \mathrm{~km}$ and same road near the Soban Gali leads to adjunct areas of Union Council Chamhad and at the end its boundary attached with the District Haripur. While on the other side the Lower Tanawal area is attached from the Sherwan side with Mansehra and from the NE it close with Tarbela lake. The climate of the lower Tanawal area is cold during winters and mild during summers with humid temperatures during June and July. During the winter, the temperature may drop to below $0^{\circ} \mathrm{C}$ and snowfall is common especially in January (Gazetteer of Hazara, 2000). The January and February is the coldest months and March and April are pleasant while May and June are hot \& dry month (Muhammad Rafique, 1997). Most rainfall occurs during the monsoon season in summer stretching from May to August and can sometimes cause flooding. 


\section{Field work and plant collection}

Extensive field surveys were carried out during 2016 to 2018. The study area was visited frequently for collection of data. The tools used during field survey were Camera (Nikon Cooplix A-100), twig Cutter, trowel, newspaper, plant presser, and polythene bags. Data was recorded in the field note book and every specimen was tagged. Specimens of each plant species have been collected, dried and fixed on standard herbarium sheets. The plant specimens were identified with the help of available literature herbarium specimen and flora of Pakistan (Nasir \& Ali 1970-1989). These specimens were then placed in Herbarium, Department of Botany Hazara University Mansehra, Pakistan.

\section{Results}

In total, 286 plant species belonging to 221 genera and 86 families were recorded from the study area (Table 1). Out of these 286 species, 281 species belonged to Angiosperms, followed by Pteridophytes with 4 species, single Gymnospermic species (Figure 1). Angiospermatophyta contains 82 families, Gymnosperm contain 1 families and Pteridophytes contains 3 families (Figure 2). In which Herbaceous growth form dominates the study area with 187 plant species followed by shrub with 54 plant species and 45 trees (Figure 3). Among all 86 families, Asteraceae was found to be the most dominant family with 25 plant species, followed by Fabaceae with 24 species followed by Rosaceae with 20 species, (Figure 4) Lamiaceae with 19 species, Poceae with 15 species respectively. (Table 1 ).

Table 1. List of plants species, biological spectrum of Lower Tanawal District Abbottabad, KP, and Pakistan.

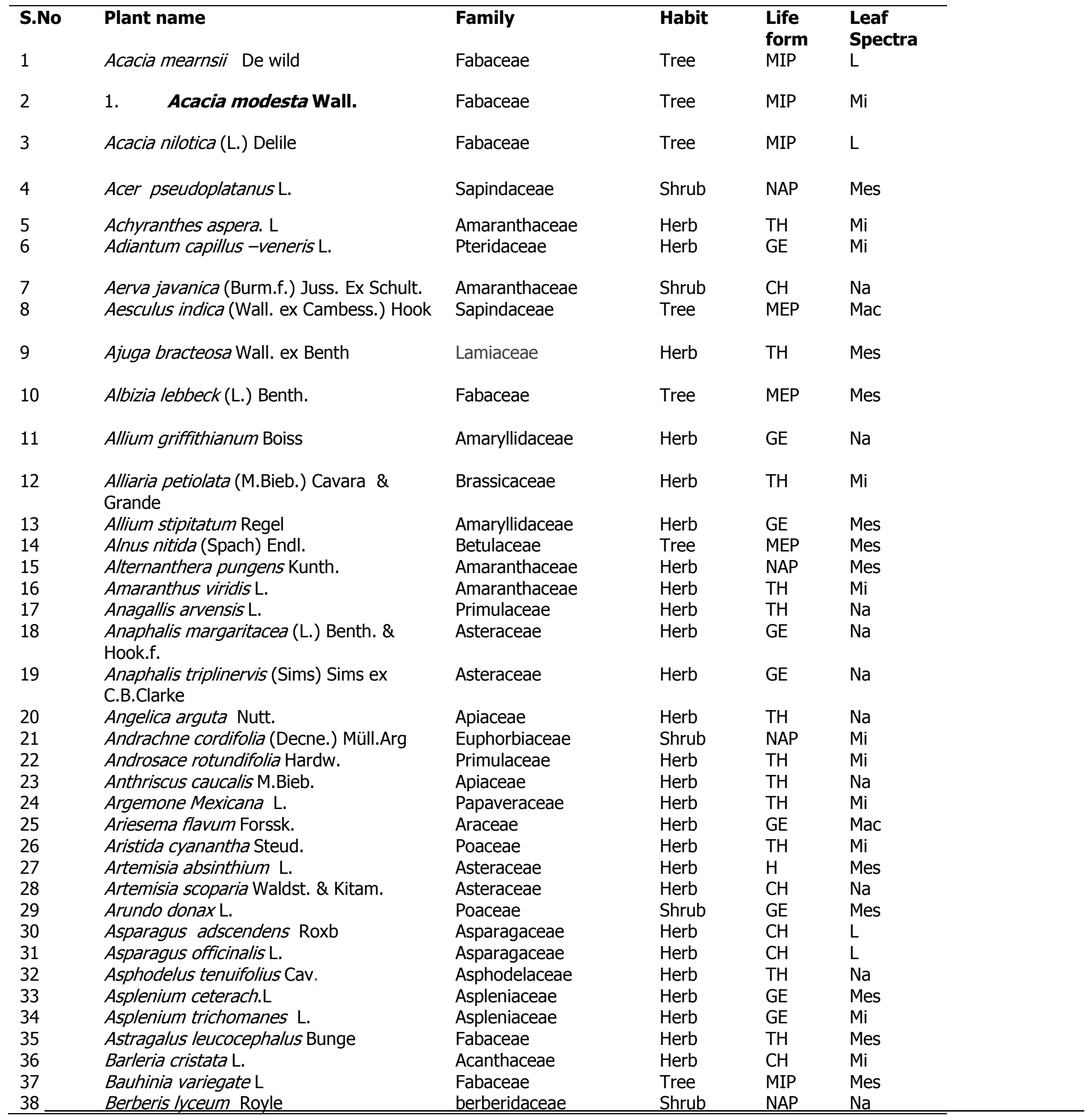




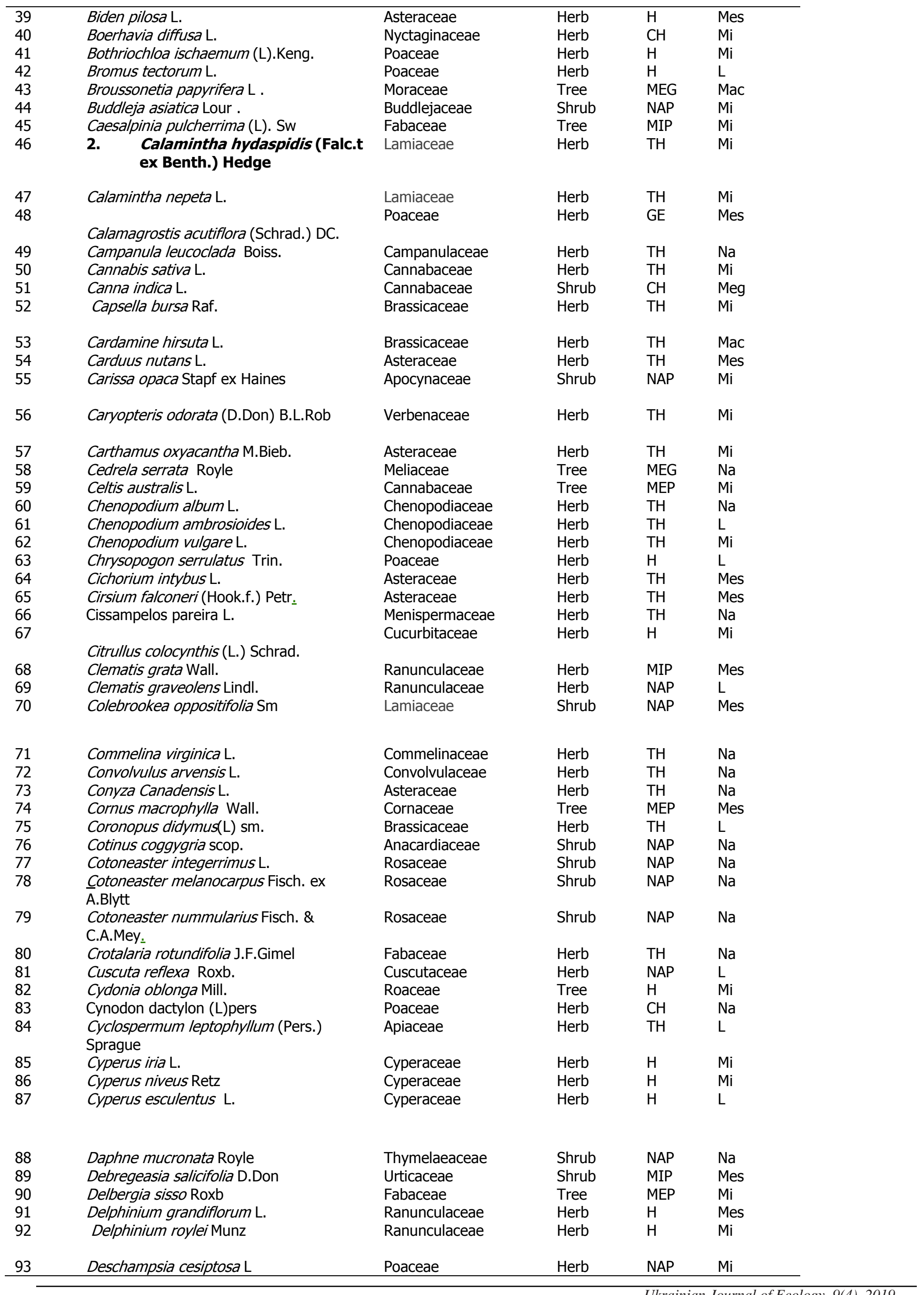




\begin{tabular}{ll}
\hline 94 & Desmodium elegan DC \\
95 & Desmodium gangeticum L \\
96 & Desmodium tortuosum (Sw.) DC. \\
97 & Dicliptera bupleuroides Nees \\
98 & Digera muricata (L).Mart. \\
99 & Diospyros lotus L \\
100 & Dodonaea viscosa (L) Jacq \\
101 & Duchesnea indica (Jacks.) Focke \\
102 & Dryopteris marginalis (L)A.Gray. \\
103 & Echinops echinatus Roxb \\
104 & Elaeagnus umbellata Thumb \\
105 & Eleusine indica (L).Gaertn. \\
106 & Eremostachys superba Royle.ex.Benth. \\
107 & Eriobotrya japonica (Thunb.)Lindl
\end{tabular}

Fabaceae
Fabaceae
Fabaceae
Acanthaceae
Amaranthaceae
Ebenaceae
Sapindaceae
Rosaceae
Dryopteriaceae
Asteraceae
Elaeagnaceae
Poaceae
Lamiaceae
Rosaceae

$\begin{array}{lll}\text { Shrub } & \text { NAP } & \text { Mes } \\ \text { Shrub } & \text { NAP } & \text { Mes } \\ \text { Shrub } & \text { NAP } & \text { Mi } \\ \text { Herb } & \text { TH } & \text { Mi } \\ \text { Herb } & \text { TH } & \text { Mi } \\ \text { Tree } & \text { MEP } & \text { Mes } \\ \text { Shrub } & \text { NAP } & \text { Mi } \\ \text { Herb } & \text { CH } & \text { Mes } \\ \text { Herb } & \text { GE } & \text { Mes } \\ \text { Shrub } & \text { CH } & \text { Mes } \\ \text { Shrub } & \text { NAP } & \text { Mi } \\ \text { Herb } & \text { H } & \text { L } \\ \text { Herb } & \text { MEP } & \text { L } \\ \text { Tree } & \text { MEP } & \text { Mac }\end{array}$

$\begin{array}{ll}108 & \text { Eryngium billardieri L. } \\ 109 & \text { Eucalyptus globulus Labill } \\ 110 & \text { Euphorbia hirta L. } \\ 111 & \text { Euphorbia peplus L. } \\ 112 & \text { Ficus benghalensis L. } \\ 113 & \text { Ficus palmata Forssk } \\ 114 & \text { Ficus recemosa L. } \\ 115 & \text { Fumaria indica (Hausskn.) Pugsley } \\ 116 & \text { Galium aparine L. } \\ 117 & \text { Galium circaezans Michx } \\ 118 & \text { Galium spurium L. } \\ 119 & \text { Galium triflorumMichx } \\ 120 & \text { Gentiana argentea (Royle ex D.Don) } \\ & \text { Royle ex D.Don } \\ 121 & \text { Geranium ocellatum Jacquem. ex } \\ & \text { Cambess. }\end{array}$

Umbelliferae
Myrtaceae
Euphorbiaceae
Euphorbiaceae
Moraceae
Moraceae
Moraceae
Fumariaceae
Rubiaceae
Rubiaceae
Rubiaceae
Rubiaceae
Gentianaceae

$\begin{array}{lll}\text { Herb } & \mathrm{H} & \mathrm{Na} \\ \text { Tree } & \mathrm{MEP} & \mathrm{Mi} \\ \text { Herb } & \mathrm{TH} & \mathrm{Mi} \\ \text { Herb } & \text { TH } & \mathrm{L} \\ \text { Tree } & \text { MEG } & \mathrm{Mes} \\ \text { Tree } & \text { MIP } & \text { Mes } \\ \text { Tree } & \text { MIP } & \mathrm{Mes} \\ \text { Herb } & \text { TH } & \mathrm{L} \\ \text { Herb } & \text { TH } & \mathrm{Na} \\ \text { Herb } & \text { TH } & \mathrm{Na} \\ \text { Herb } & \text { TH } & \mathrm{L} \\ \text { Herb } & \text { TH } & \mathrm{Na} \\ \text { Herb } & \mathrm{H} & \mathrm{Na} \\ & & \\ \text { Herb } & \mathrm{CH} & \mathrm{Mi}\end{array}$

122 Geranium rotundifolium $\mathrm{L}$.

Geraniaceae

Herb

Geranium robertianum L.

Geraniaceae

GE

Mes

Grewia optiva J.R.Drumm. ex Burre

Tiliaceae

Herb

GE $\quad \mathrm{Na}$

Tree MIP Mes

Gymnosporia royleana Wall. ex M.A.Lawson

Celastraceae

Shrub NAP Mi

Hedera helix L.

127 Heliotropium bacciferum Forssk

Heracleum sphondylium L.

Hypericum foliosum Aiton

Araliaceae

Boraginaceae

Apiaceae

Hypericaceae

Hypericaceae

Poaceae

Balsaminaceae

Fabaceae

Fabaceae

Convolvulaceae

Convolvulaceae

Lamiaceae

Oleaceae

Juglandaceae

Acanthaceae

Acanthaceae

Scrophulariaceae

Asteraceae

Asteeaceae

Lamiaceae

Anacardiaceae

Verbenaceae

Boraginaceae

Fabaceae

Asteraceae

$\begin{array}{lll}\text { Herb } & \text { NAP } & \text { Mes } \\ \text { Herb } & \text { GE } & \text { L } \\ \text { Herb } & \text { H } & \text { Mes } \\ \text { Shrub } & \text { TH } & \text { Mi } \\ & & \\ \text { Shrub } & \text { TH } & \text { Na } \\ \text { Herb } & \text { H } & \text { Mi } \\ \text { Herb } & \text { TH } & \text { Mi } \\ \text { Shrub } & \text { NAP } & \text { Mi } \\ \text { Herb } & \text { CH } & \text { L } \\ \text { Shrub } & \text { TH } & \text { Mac } \\ \text { Herb } & \text { TH } & \text { Mi } \\ \text { Shrub } & \text { NAP } & \text { Mi } \\ \text { Herb } & \text { NAP } & \text { Mi } \\ \text { Tree } & \text { MEG } & \text { Mes } \\ \text { Shrub } & \text { NAP } & \text { Mes } \\ \text { Herb } & \text { TH } & \text { Na } \\ \text { Herb } & \text { TH } & \text { Mes } \\ \text { Herb } & \text { TH } & \text { Mes } \\ \text { Herb } & \text { TH } & \text { Mi } \\ \text { Herb } & \text { TH } & \text { Mi } \\ \text { Tree } & \text { MEG } & \text { Mi } \\ \text { Shrub } & \text { NAP } & \text { Na } \\ \text { Herb } & \text { TH } & \text { L } \\ \text { Herb } & \text { CH } & \text { Na } \\ \text { Herb } & \text { CH } & \text { Mi }\end{array}$




\begin{tabular}{|c|c|c|c|c|c|}
\hline & Rajagopal & & & & \\
\hline 151 & Lepidium didymium $\mathrm{L}$. & Brassicaceae & Herb & $\mathrm{TH}$ & $\mathrm{L}$ \\
\hline 152 & Leptodermis virgata Edgew. ex Hook.f. & Rubiaceae & Shrub & $\mathrm{CH}$ & $\mathrm{Na}$ \\
\hline 153 & Lespedza juncea Linn.f & Fabaceae & Herb & $\mathrm{TH}$ & $\mathrm{L}$ \\
\hline 154 & Linum corymbulosum Rchb & Linaceae & Herb & $\mathrm{TH}$ & $\mathrm{Na}$ \\
\hline 155 & Limonium echioides (L.) Mill. & plumbaginaceae & Herb & $\mathrm{TH}$ & $\mathrm{Na}$ \\
\hline 156 & Lonicera quinquelocularis Hard. & Carprifoliaceae & Shrub & NAP & Mi \\
\hline 157 & Malvastrum coromandelianum (L.) Garcke & Malvaceae & Herb & $\mathrm{TH}$ & Mes \\
\hline 158 & Mallotus philippensis (Lam) Muell.Arg. & Euphorbiaceae & Shrub & MIP & Mes \\
\hline 159 & 3. Malva neglecta Wallr. & Malvaceae & Herb & & \\
\hline & & & & $\mathrm{CH}$ & Mi \\
\hline 160 & Marrubium vulgare $\mathrm{L}$. & Lamiaceae & Herb & $\mathrm{CH}$ & $\mathrm{Mi}$ \\
\hline 161 & Melia azedarach L. & Meliaceae & Tree & MIP & Mes \\
\hline 162 & Medicago denticulata Willd. & Fabaceae & Herb & $\mathrm{TH}$ & $\mathrm{Na}$ \\
\hline 163 & Medicago polymorpha L. & Fabaceae & Herb & $\mathrm{TH}$ & $\mathrm{Na}$ \\
\hline 164 & Melilotus indicus( L).All. & Fabaceae & Herb & $\mathrm{TH}$ & $\mathrm{Na}$ \\
\hline 165 & Mentha arvensis $\mathrm{L}$. & Lamiaceae & Herb & GE & Mi \\
\hline 166 & Menthe longifolia(L)Huds. & Lamiaceae & Herb & GE & $\mathrm{Mi}$ \\
\hline 167 & $\begin{array}{l}\text { Micromeria biflora (Buch.-Ham. ex } \\
\text { D.Don) Benth. }\end{array}$ & Lamiaceae & Herb & $\mathrm{TH}$ & $\mathrm{L}$ \\
\hline 168 & Mirabilis jalapa L. & Nyctaginaceae & Herb & $\mathrm{CH}$ & Mes \\
\hline 169 & Morus alba L. & Moraceae & Tree & MIP & Mes \\
\hline 170 & Morus nigra L. & Moraceae & Tree & MIP & Mes \\
\hline 171 & Myrsine Africana L. & Primulaceae & Shrub & NAP & $\mathrm{Na}$ \\
\hline 172 & Nasturtium officinale R.Br. & Brassicaceae & Herb & $\mathrm{TH}$ & $\mathrm{Na}$ \\
\hline 173 & $\begin{array}{l}\text { Nanorrhinum acerbianum (Boiss.) } \\
\text { Betsche }\end{array}$ & Plantaginaceae & Herb & $\mathrm{TH}$ & $\mathrm{Na}$ \\
\hline 174 & Nannorrhop ritchiana(Griff) .Aitchison. & Arecaceae & Shrub & MIP & Mes \\
\hline 175 & Nepeta cataria L. & Lamiaceae & Herb & $\mathrm{CH}$ & Mi \\
\hline 176 & Nepeta ciliaris Benth & Lamiaceae & Herb & $\mathrm{TH}$ & Mi \\
\hline 177 & Notholirion thomsonianum(Royle)stapf & Liliaceae & Herb & GE & Mi \\
\hline 178 & Oenothera rosea L. & Onagraceae & Herb & $\mathrm{TH}$ & $\mathrm{Na}$ \\
\hline 179 & Olea ferruginea Royle. & Oleaceae & Tree & MIP & $\mathrm{Na}$ \\
\hline 180 & Onosma hispida Wall. ex G. Don & Boraginaceae & Herb & $\mathrm{TH}$ & Mic \\
\hline 181 & Opuntia ovata Pfeiff. & Cactaceae & Shrub & NAP & Mac \\
\hline 182 & Origanum vulgare L. & Lamiaceae & Herb & $\mathrm{TH}$ & $\mathrm{Na}$ \\
\hline 183 & Otostegia limbata(Benth).Boiss & Lamiaceae & Shrub & NAP & Mi \\
\hline 184 & Oxalis corniculata( $(\mathrm{L})$ & Oxalidaceae & Herb & $\mathrm{TH}$ & $\mathrm{Na}$ \\
\hline 185 & Parthenium hysterophous $\mathrm{L}$. & Asteraceae & Herb & $\mathrm{TH}$ & Mes \\
\hline 186 & Pennisetum orientale .Rich & Poaceae & Herb & $\mathrm{TH}$ & Mi \\
\hline 187 & Pentanema vestitum Wall .ex. DC & Asteraceae & Herb & $\mathrm{H}$ & Mes \\
\hline 188 & Periploca aphylla.Decne. & Apocynaceae & Shrub & NAP & Mac \\
\hline 189 & Perilla frutescens(L) Britton. & Lamiaceae & Herb & $\mathrm{TH}$ & Mi \\
\hline 190 & Phleum alpinum $\mathrm{L}$. & Poaceae & Herb & $\mathrm{H}$ & $\mathrm{Na}$ \\
\hline 191 & Phleum arenarium $\mathrm{L}$. & Poaceae & Herb & $\mathrm{H}$ & $\mathrm{Na}$ \\
\hline 192 & Pimpinella stewartii Nasir & Apiaceae & Herb & $\mathrm{TH}$ & $\mathrm{Na}$ \\
\hline 193 & Pinus roxburghii Sarg. & Pinaceae & Tree & MEG & $\mathrm{Na}$ \\
\hline 194 & $\begin{array}{l}\text { Pistacia integerrima } \\
\text { J.L.Stewart.ex.Brands. }\end{array}$ & Anacardiaceae & Tree & MEP & Mac \\
\hline 195 & Plantago lanceolata L. & Plantaginaceae & Herb & $\mathrm{TH}$ & Mi \\
\hline 196 & Plumbago zeylanica $\mathrm{L}$. & plumbaginaceae & Herb & $\mathrm{TH}$ & $\mathrm{Mi}$ \\
\hline 197 & Poa balfourii auct. & Poaceae & Herb & $\mathrm{TH}$ & $\mathrm{Na}$ \\
\hline 198 & Poa poiformis Labill & Poaceae & Herb & $\mathrm{TH}$ & $\mathrm{L}$ \\
\hline 199 & Polygala abyssinica R.Br.ex.Fresen. & Polygalaceae & Herb & $\mathrm{CH}$ & $\mathrm{Na}$ \\
\hline 200 & Polygala vulgaris L. & Polygalaceae & Herb & $\mathrm{H}$ & Mi \\
\hline 201 & Polygonum plebeium R.Br. & Polygonaceae & Herb & $\mathrm{H}$ & $\mathrm{L}$ \\
\hline 202 & Polygonum viviparum L. & Polygonaceae & Herb & $\mathrm{TH}$ & $\mathrm{L}$ \\
\hline 203 & Populous alba L. & Salicaceae & Tree & MEG & Mes \\
\hline 204 & Potentilla rivalis Nutt. ex Torr. \& A.Gray & Rosaceae & Herb & $\mathrm{TH}$ & $\mathrm{Mi}$ \\
\hline 205 & Prunus amricana Marsh & Rosaceae & Tree & MIP & $\mathrm{Mi}$ \\
\hline 206 & Prunus domestica L. & Rosaceae & Tree & MIP & Mes \\
\hline 207 & Prunus persica(L).Batsch. & Rosaceae & Tree & MIP & Mi \\
\hline 208 & Psidium guajava L. & Myrtaceae & Tree & MIP & Mes \\
\hline 209 & Punica granatum $\mathrm{L}$. & Punicaceae & Tree & MIP & $\mathrm{Na}$ \\
\hline
\end{tabular}




\begin{tabular}{|c|c|c|c|c|c|}
\hline 210 & Pyrus pashia Buch.-Ham. ex D.Don & Rosaceae & Tree & MIP & Mi \\
\hline 211 & Pyrus pyrifolia(Burm).Nak. & Rosaceae & Tree & MIP & Mes \\
\hline 212 & Pyrus communis $\mathrm{L}$. & Rosaceae & Tree & MIP & Mes \\
\hline 213 & Quercus incana Bartram & Fagaceae & Tree & MEG & Mes \\
\hline 214 & $\begin{array}{l}\text { Randia tetrasperma (Wall. ex Roxb.) } \\
\text { Benth. }\end{array}$ & Rubiaceae & Shrub & $\mathrm{TH}$ & $\mathrm{Na}$ \\
\hline 215 & Ranunculus muricatus $\mathrm{L}$. & Ranunculaceae & Herb & $\mathrm{TH}$ & Mi \\
\hline 216 & Ranunculus parviflorus L. & Ranunculaceae & Herb & $\mathrm{TH}$ & $\mathrm{Mi}$ \\
\hline 217 & Reinwardtia trigyna Planch. & Linaceae & Herb & NAP & $\mathrm{Na}$ \\
\hline 218 & Rhamnus virgata Roxb. & Rhamnaceae & Shrub & MIP & Mi \\
\hline 219 & Rhynchosia tomentosa(L)Hook \& Arn. & Fabaceae & Herb & NAP & $\mathrm{L}$ \\
\hline 220 & Ricinus communis $\mathrm{L}$. & Euphorbiaceae & Tree & MIP & Mac \\
\hline 221 & Rosa brunonii L. & Rosaceae & Shrub & NAP & $\mathrm{Na}$ \\
\hline 222 & Rosa moschata(Herrm) & Rosaceae & Shrub & MIP & Mes \\
\hline 223 & Robina pseudoacacia $\mathrm{L}$ & Fabaceae & Tree & MEG & Mes \\
\hline 224 & Rubia cordifolia L. & Rubiaceae & Herb & $\mathrm{TH}$ & $\mathrm{Na}$ \\
\hline 225 & Rubia manjith Roxb & Rubiaceae & Herb & $\mathrm{TH}$ & $\mathrm{Na}$ \\
\hline 226 & Rubus ellipticus Sm. & Rosaceae & Shrub & NAP & Mes \\
\hline 227 & Rubus fruticosus $\mathrm{L}$. & Rosaceae & Shrub & NAP & Mes \\
\hline 228 & Rubus sanctus Schreb. & Rosaceae & Shrub & NAP & $\mathrm{Mi}$ \\
\hline 229 & Rumex dentatus $\mathrm{L}$. & Polygonaceae & Herb & GE & Mes \\
\hline 230 & Rumex hastatus D.Don. & polygonaceae & Herb & NAP & Mes \\
\hline 231 & Rumex nepalensis Spreng & Polygonaceae & Herb & GE & Mes \\
\hline 232 & Salix acmophylla Boiss & Salicaceae & Tree & MIP & $\mathrm{Mi}$ \\
\hline 233 & Salvia coccinea Buc'hoz ex Etl. & Lamiaceae & Herb & $\mathrm{CH}$ & $\mathrm{Mi}$ \\
\hline 234 & Salvia moorcroftiana Wall. ex Benth. & Lamiaceae & Herb & $\mathrm{CH}$ & Mes \\
\hline 235 & Sapindus mukorossi Gaertn & Sapindaceae & Tree & MEG & Mes \\
\hline 236 & Saussurea heteromalla D.Don & Asteraceae & Herb & $\mathrm{TH}$ & $\mathrm{Mi}$ \\
\hline 237 & Scabiosa candollei Wall. & Dipsaceae & Herb & $\mathrm{H}$ & Mes \\
\hline 238 & Scabiosa ochroleuca L. & Caprifoliaceae & Herb & TH & $\mathrm{Na}$ \\
\hline 239 & Scandix pectin -veneris $\mathrm{L}$. & Apiaceae & Herb & TH & $\mathrm{Mi}$ \\
\hline 240 & Scilla griffithii Hochr. & Hyacinthaceae & Herb & GE & $\mathrm{Na}$ \\
\hline 241 & Scrophularia dentata Royle .ex.Bentham. & Scrophulariaceae & Herb & $\mathrm{TH}$ & $\mathrm{Na}$ \\
\hline 242 & Sedum sarmentosum Bunge & Crassulaceae & Herb & GE & $\mathrm{Na}$ \\
\hline 243 & Senecio aquaticus Hill. & Asteraceae & Herb & TH & Mes \\
\hline 244 & Sida cordata (Burm.f.) Borss. Waalk & Malvaceae & Herb & TH & $\mathrm{Mi}$ \\
\hline 245 & Sisymbrium irio $\mathrm{L}$. & Brassicaceae & Herb & TH & $\mathrm{Na}$ \\
\hline 246 & Silene conoidea $\mathrm{L}$. & Caryophyllaceae & Herb & TH & $\mathrm{Na}$ \\
\hline 247 & Smilax -bona-nox L. & Smilacaceae & Herb & TH & $\mathrm{L}$ \\
\hline 248 & Smilax china L. & Smilacaceae & Herb & TH & $\mathrm{Na}$ \\
\hline 249 & Smilax rotundifolia L. & Smilacaceae & Herb & TH & $\mathrm{Na}$ \\
\hline 250 & Solanum erianthum D.Don & Solanaceae & Herb & TH & Mes \\
\hline 251 & Solanum incanum $\mathrm{L}$. & Solanaceae & Herb & $\mathrm{CH}$ & Mes \\
\hline 252 & Solanum nigrum $\mathrm{L}$. & Solanaceae & Herb & $\mathrm{TH}$ & Mes \\
\hline 253 & Solanum surattense Burm. f. & Solanaceae & Herb & $\mathrm{CH}$ & Mes \\
\hline 254 & Sonchus asper (L). Hill & Asteraceae & Herb & $\mathrm{CH}$ & $\mathrm{Mi}$ \\
\hline 255 & Sonchus oleracus (L.)Hill & Asteraceae & Herb & MIP & Mes \\
\hline 256 & Sorbaria tomentosa (Lindl.) Rehder & Rosaceae & Shrub & MIP & Mes \\
\hline 257 & Stachys emodi.Hedge. & Lamiaceae & Herb & TH & $\mathrm{Mi}$ \\
\hline 258 & Spiraea vaccinifolia D.Don & Rosaceae & Shrub & NAP & $\mathrm{Mi}$ \\
\hline 259 & Stellaria alsinoides Boiss & Caryophyllaceae & Herb & $\mathrm{TH}$ & L \\
\hline 260 & Stellaria media (L)Vill. & Caryophyllaceae & Herb & $\mathrm{CH}$ & $\mathrm{Na}$ \\
\hline 261 & Strobilanthes efloraofindia C.B.Clarke & Acanthaceae & Herb & TH & $\mathrm{Na}$ \\
\hline 262 & Strobilanthes oliganthus(Miq) & Acanthaceae & Herb & TH & $\mathrm{Na}$ \\
\hline 263 & Symplocos recemosa Roxb & Symplocaceae & Tree & TH & Mes \\
\hline 264 & Tagetes minuta $\mathrm{L}$. & Asteraceae & Herb & TH & Mes \\
\hline 265 & Taraxacum officinale L & Asteraceae & Herb & TH & Mes \\
\hline 266 & Tricholepis angustifolia-DC & Asteraceae & Herb & TH & $\mathrm{Mi}$ \\
\hline 267 & Trichodesma indicum $(\mathrm{L}) \mathrm{R} . \mathrm{Br}$ & Boraginaceae & Herb & TH & $\mathrm{Na}$ \\
\hline 268 & Tridax procumbens $\mathrm{L}$. & Asteraceae & Herb & $\mathrm{H}$ & $\mathrm{Mi}$ \\
\hline 269 & Trifolium repen L. & Fabaceae & Herb & GE & $\mathrm{Na}$ \\
\hline 270 & Ulmus villosa Brandes.ex.gamble & Ulmaceae & Tree & MEG & $\mathrm{Mi}$ \\
\hline 271 & Verbena officinalis $\mathrm{L}$ & Verbenaceae & Herb & $\mathrm{TH}$ & $\mathrm{Mi}$ \\
\hline 272 & Veronica stewartii Pennel & Plantaqinaceae & Herb & TH & $\mathrm{Na}$ \\
\hline 273 & Verbena tenuisecta Briq. & Verbenaceae & Herb & TH & $\mathrm{Na}$ \\
\hline 274 & Verbascum Thapsus L. & Scrophulariaceae & Herb & $\mathrm{H}$ & Mes \\
\hline 275 & Viburnum cotinifolium D.Don & Caprifoliaceae & Shrub & MIP & Mes \\
\hline
\end{tabular}




\begin{tabular}{|c|c|c|c|c|c|}
\hline 276 & Vicia hirsuta(L)Gray. & Fabaceae & Herb & $\mathrm{TH}$ & $\mathrm{Na}$ \\
\hline 277 & Vicia sativa $\mathrm{L}$. & Fabaceae & Herb & $\mathrm{TH}$ & $\mathrm{Na}$ \\
\hline 278 & Viola Odorata L & Violaceae & Herb & $\mathrm{TH}$ & Mes \\
\hline 279 & Vitex negundo L. & Verbenaceae & Shrub & NAP & Mes \\
\hline 280 & Vitis vinifera $\mathrm{L}$. & Vitaceae & Herb & NAP & Mes \\
\hline 281 & Woodfordia fruticosa( L.)Kurz. & Lythraceae & Shrub & NAP & Mes \\
\hline 282 & Xanthium strumarium $\mathrm{L}$. & Asteraceae & Shrub & $\mathrm{TH}$ & Mes \\
\hline 283 & Zanthoxylum armatum Dc. & Rutaceae & Shrub & MIP & Mes \\
\hline 284 & Ziziphus oenopila L. & Rhamnaceae & Shrub & $\mathrm{CH}$ & $\mathrm{Na}$ \\
\hline 285 & $\begin{array}{l}\text { Ziziphus nummularia (Burm.f.) Wight \& } \\
\text { Arn. }\end{array}$ & Rhamnaceae & Shrub & NAP & Mi \\
\hline 286 & Ziziphus jujuba Mill. & Rhamnaceae & Shrub & NAP & Mi \\
\hline
\end{tabular}

$\mathrm{TH}=$ Therophytes,MEP=Mesophenrophytes,MEG=Megaphanerophytes,MIP=Microphenerophytes, $\mathrm{HC}=$ Hemicryptophytes, $\mathrm{NAP}=$ Nanophanerophytes, GE=Geophytes, CH=Chemeophytes, Mi=Microphyll, Mes=Mesophyll, L=Leptpphyll, Na=Nanophyll, Mac=Macrophyll, Meg=Megaphyll.

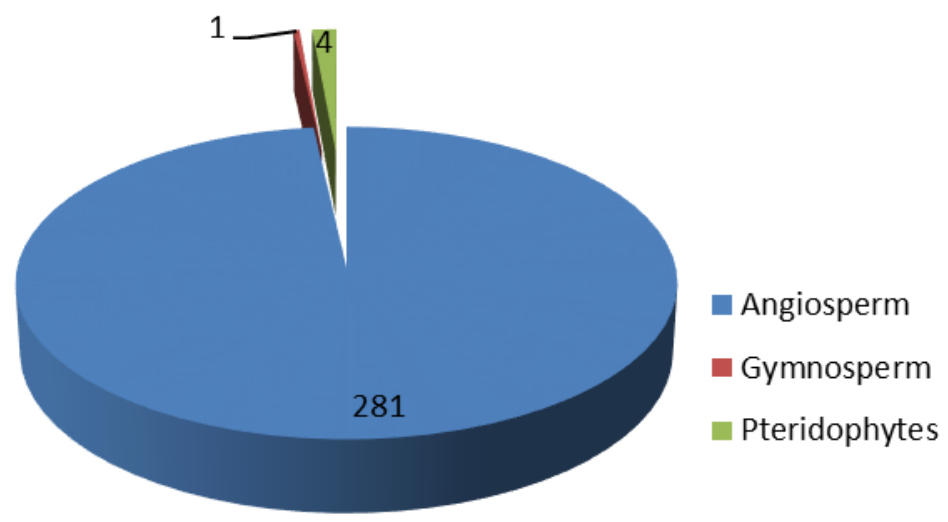

Figure 1. Division of plant species recorded from study area.

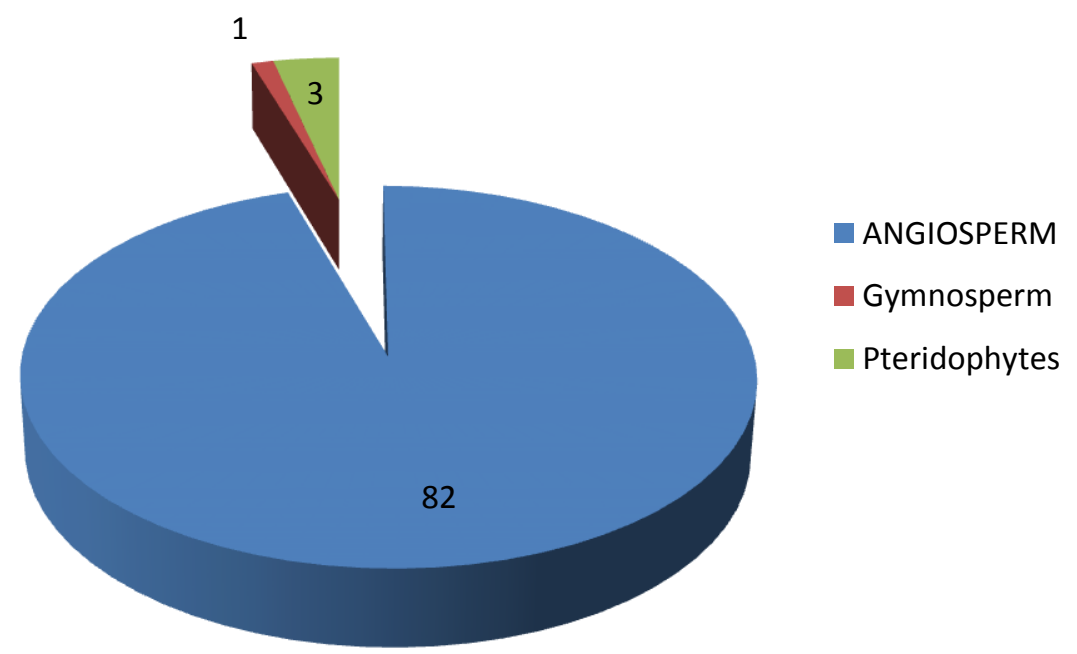

Figure 2. Angiosperm, gymnosperms and pteridophytes families of plant species recorded from study area. 


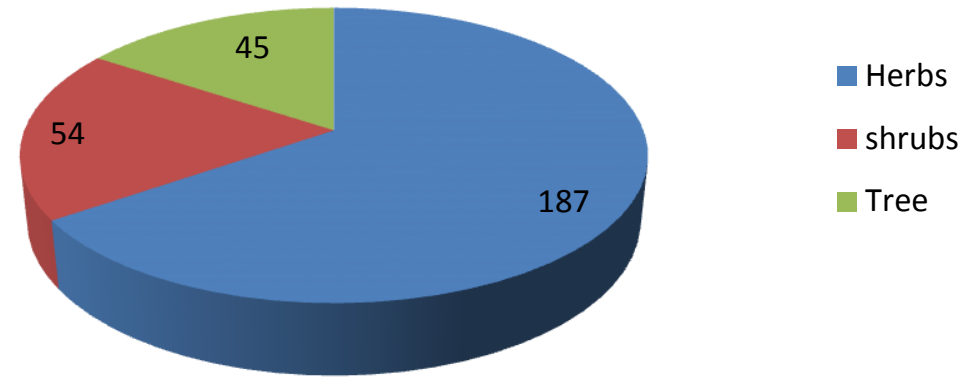

Figure 3. Growth habit of plant species recorded from the study area.

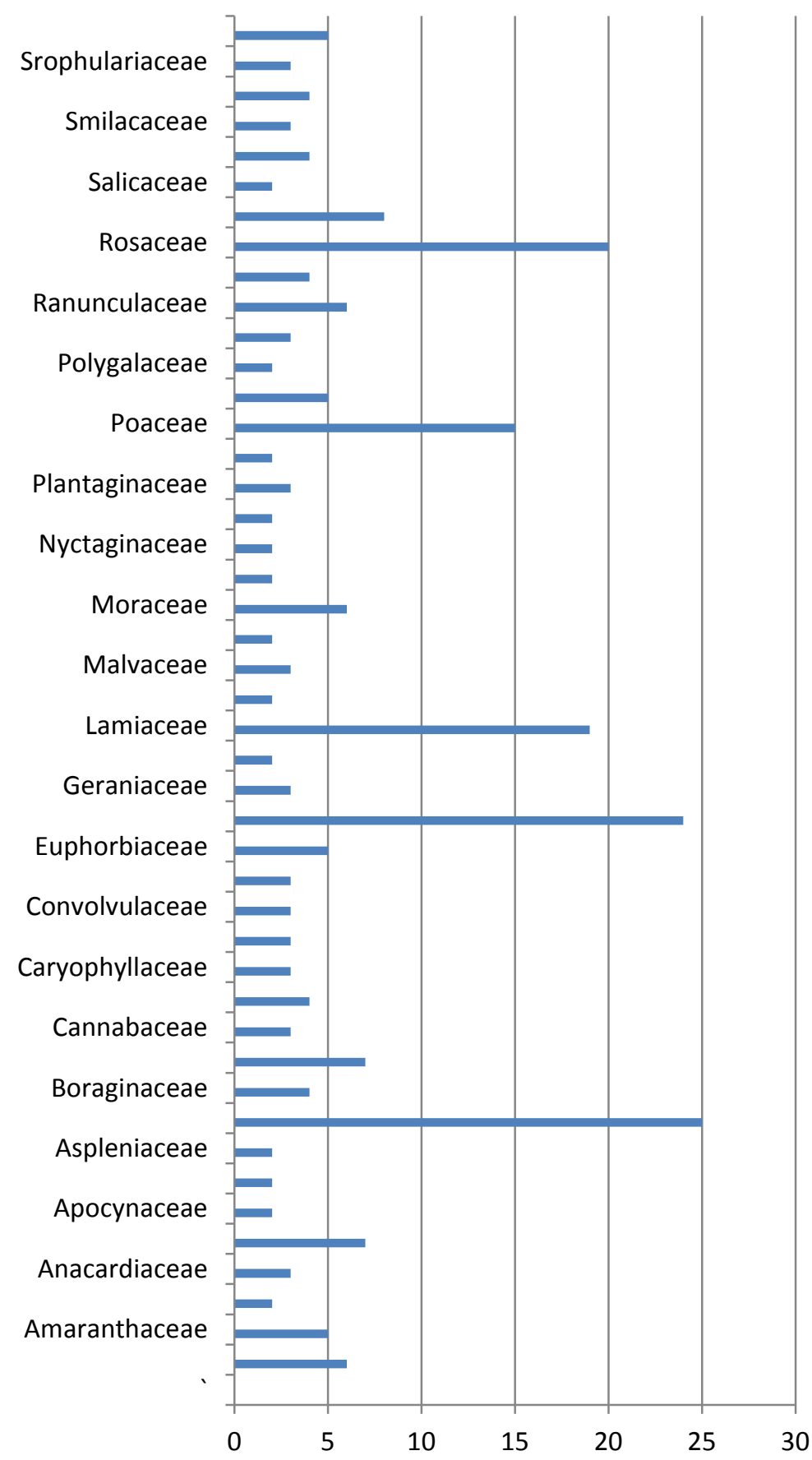

Figure 4. Distribution of plants family having two or more than two plant species. 
Life form classes and leaf spectra were determined by Raunkier's method By using this method seven life form classes are observed in which Therophytes was most dominant with 113 species (39.5104\%),followed by Nanophanerophytes with 45 species (15.7342\%),then Microphanerophytes with 32 species (11.188\%), Chamaeophytes with 27 species (9.4405\%),Hemicryptophytes with 25 species (8.7412\%) Geophytes with 22 species (7.69\%) followed by Megaphanerophytes and Mesophanerophytes with 11 species (3.8461\%) each (Figure 5). In leaf size spectra, Microphyll are dominants with 88 species (30.7692\%) followed by Mesophyll with 81 species (28.32\%) Nanophyll with 77 species (26.9230\%) Leptophyll with 29 species (10.13\%) Macrophyll with 10 species (3.49\%) and Megaphyll with 1 species (0.3496\%) (Figure 6).

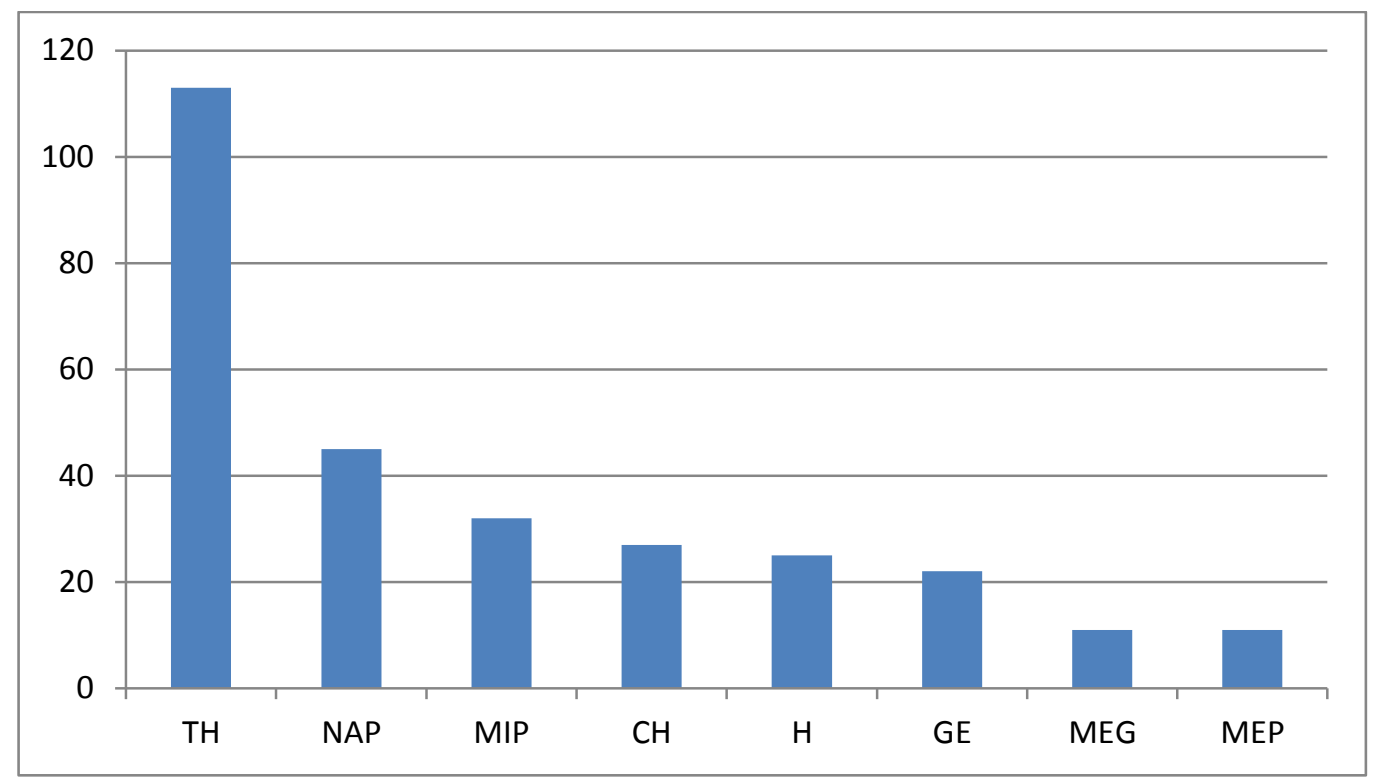

Figure 5. Graphical representation of life form classes of Lower Tanawal.

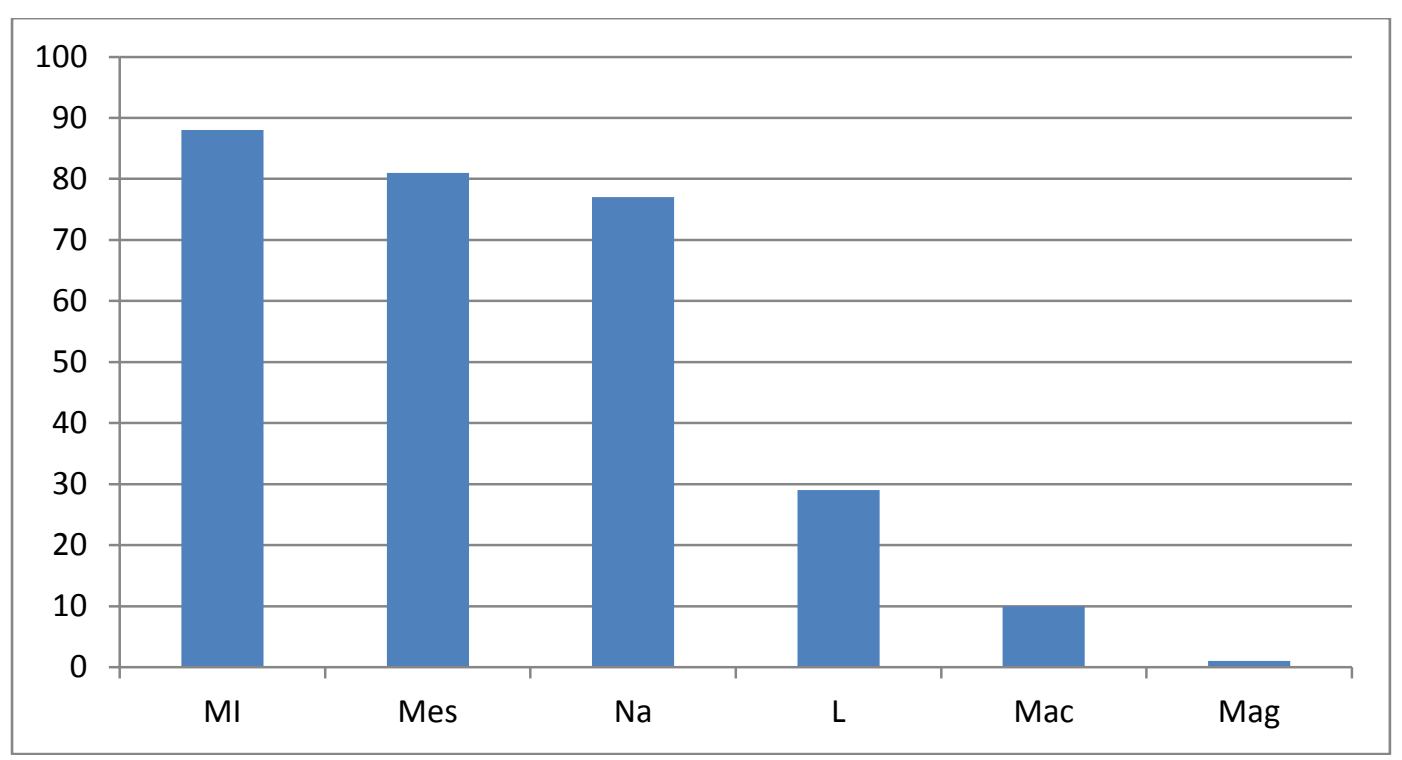

Figure 6. Graphical representation of leaf spectra of Lower Tanawal.

\section{Discussion}

This research study reveal that previously no such study has been conducted on floristic composition of Lower Tanawal as well as no proper documentation is present on floristic composition of Lower Tanawal. Thus, our study provides complete checklist of flora of Lower Tanawal. Biological Spectra indicate the prevailing environmental condition of any area (Hussain et al. 2015). Life form classes are very important to determine the plant communities in a study area. Disturbance such as deforestation, extensive grazing and fire and biotic pressure effect plant life form, phenology. In our study area biological spectrum showed that out of 286 Species Therophytes was most dominant with 113 species (39.5104\%),followed by Nanophanerophytes with 45 species (15.7342\%),then Microphanerophytes with 32 species $(11.188 \%)$, Chamaephytes with 27 species $(9.4405 \%)$, Hemicryptophytes with 25 species $(8.7412 \%)$.Geophytes with 22 species $(7.6923 \%)$,followed by Megaphanerophytes \& Mesophanerophytes with 11 species (3.8461\%)each (Figure 5). Therophytes was dominant life form which indicates disturbed environmental conditions in the study area, and biotic pressure on vegetation which increase the short live species. Our findings are in agreement with Saxena et al. (2004); Farag (2014) and Ali et al. (2016) which show that biotic pressure effect the vegetation. As Costa et al. (2007) reported that therophytes are dominant life form in South Eastern Brazil which is similar to our finding. Fazal et al. (2010) dominancy of therophytes show degraded and unfavorable environmental condition. Our results are agreed with the finding of Al Yemen's and Sher (2010). In leaf size spectra, Microphyll are dominants with 88 species $(30.7692 \%)$, followed by Mesophyll with 81 species 
(28.3216\%), Nanophyll with 77 species (26.9230\%), Leptophyll with 29 species (10.1398\%), Macrophyll with 10 species (3.4965\%), and Megaphyll with 1 species (Figure 6).

\section{References}

Ali, S.I., Nasir, Y.J. (1989). Flora of Pakistan. Nos. 191-193. Islamabad, Karachi.

Ali, S.I., Qaiser, M. (1993). Flora of Pakistan. No. 194-216. Karachi.

Ali, A., Badshah, L., Hussain, F., Shinwari., Z. K. (2016). Floristic composition and ecological characteristics of plants of Chail Valley, District Swat, Pakistan. Pak. J. Bot. 48(3): 1013-1026.

Al-Yemeni, M.N., Sher, H. (2010). Biological spectrum with some others ecological attributes of the flora and vegetation of the Asir Mountain of South West, Saudi Arabia. Afr. J. Biotechnol. 9 (34): 5556-5565.

Costa, R.C., Araujo., F. S., Lima-Verde, L. W. (2007). Flora and life-form spectrum in an area of deciduous thorn woodland

Caatinga) in Northeastern, Brazil. J. Arid Environments, 68(2): 237-247.

Fazal, H., Ahmad, N., Rashid, A., Farooq, S. (2010). A checklist of phanerogamic flora of Haripur Hazara, Khyber Pakhtunkhwa, Pakistan. Pak J Bot, 42(3), 1511-1522.

Farag, El. M. (2014). Floristic Composition and traditional uses of plant species at Wadi Alkuf, Al-Jabal Al-Akhder, Libya.AmericanEurasian Journal of Agricultural \& Environmental Sciences. 14, 685-697.

Gazetteer of the Hazara District 1883-4,Lahore Sang-e-Meel Publications, 2000.

Hussain, F., Shah, S. M., Badshah, L., Durrani, M. J. (2015). Diversity and ecological characteristics of flora of Mastuj valley, district Chitral, Hindukush range, Pakistan. Pak. J. Bot, 47(2), 495-510.

Iqbal, M., Khan, S., Khan, M. A., Rahman, I. U., Abbas, Z., Zahidullah. (2015). Exploration and inventorying of weeds in wheat crop of the district Malakand, Pakistan. Pak. J. Weed Sci. Res., 21(3): 435- 452.

Ijaz, F., Iqbal, Z., Alam, J., Khan, S.M., Afzal, A., Rahman, I.U., Afzal, M., Islam, M., Sohail. (2015). Ethnomedicinal study upon folk recipes against various human diseases in Sarban Hills, Abbottabad, Pakistan. World J Zoology, 10(1): 41-46.

Ijaz, F., Iqbal, Z., Rahman, I.U., Khan, S.M., Shah, G.M., Khan, K., Afzal, A. (2016). Investigation of traditional medicinal floral knowledge of Sarban Hills, Abbottabad, KP, Pakistan. J Ethnopharmacol

Khattak, N.S., Nouroz, F., Rahman, I. U., Noreen, S. (2015). Ethno veterinary uses of medicinal plants ofdistrict Karak, Pakistan. J. Ethnopharmacol. 171: 273-279.

Muhammad, S., Hussain, M., Rahman, I. U., Shah, G. M., Ijaz, F., Ullah, K. (2016). Indigenous medicinal usage of family Asteraceae in Sadda Lower Kurram Agency: A Case Study. Asian J. Sci. \& Technol. 7(12): 3998-4003.

Muhammad., Qasim., Hanif. (2014). Stratigraphic characterization of the Early Cambrian Abbottabad Formation in the Sherwan area, Hazara region, N. Pakistan: Implications for Early Paleozoic stratigraphic correlation in NW Himalayas, Pakistan Journal of Himalayan Earth Sciences 47(1),25-40.

Nasir, E., Ali, S. I. (1970). Flora of West Pakistan. 1-131. Islamabad, Karachi.

Nasir, E., Ali, S. I. (1980). Flora of Pakistan. No. 132-190. Islamabad, Karachi.

Rahman, I.U., Ijaz, F., Iqbal, Z., Afzal, A., Ali, N., Afzal, M., Khan, M.A., Muhammad, S., Qadir, G., Asif, M. (2016). A novel survey of the ethno medicinal knowledge of dental problems in Manoor Valley(Northern Himalaya), Pakistan J Ethnopharmacol. 194C, 877894.

Rahman, I. U., Ijaz, F. A., Afzal, Z., Iqbal, N., Ali, Khan, S. M. (2016). Contributions to the phytotherapiesof digestive disorders; Traditional knowledge and cultural drivers of Manoor Valley, Northern Pakistan. J.Ethnopharmacol. 192, 30-52.

Raunkiaer, C. (1934). The Life forms of plants and statistical plant geography, Oxford.

Rafique, M. (1996).Galis Guzara Forests N.W.F.P.Forestry pre-investment centre Peshawar.

Shaheen, S., Iqbal, Z., Ijaz, F., Alam, J., Rahman, I. U. (2016). Floristic composition, biological spectrum and phenology of Tehsil Havelian, District Abbottabad, Pakistan. Pak. J. Bot. 48(5): 1849-1859

Shah, A.H., Khan, S. M., Shah, A. H., Mehmood, A., Rahman, I. U., Ahmad, H. (2015). Cultural uses of plants among Basikhel Tribe of District Tor Ghar, Khyber Pakhtunkhwa, Pakistan. Pak. J. Bot., 47(SI): 23-41. J. Appl. Environ. Biol. Sci., 7(6)158-168, 2017 Saxena, A.K., Pandey, P., Singh, J. S. (2004). Biological spectrum and other structural functional attributes of the vegetation of Kumaun Himalaya. Plant ecology, 49(2): 111-119.

\section{Citation:}

Adeela Bibi, Zafar Iqbal, Ghulam Mujtaba Shah, Manzoor Hussain and Inayat UR Rahman. (2019). Floristic Diversity, Biological Spectrum of Lower Tanawal, KP, Pakistan. Ukrainian Journal of Ecology, 9(4), 505-514.

(cc) $\mathrm{EY}$ This work is licensed under a Creative Commons Attribution 4.0. License 